\title{
Composite fermions in a long-range random magnetic field: Quantum Hall effect versus Shubnikov-de Haas oscillations
}

\author{
A. D. Mirlin, ${ }^{1, *}$ D. G. Polyakov, ${ }^{2, \dagger}$ and P. Wölfle ${ }^{1}$ \\ ${ }^{1}$ Institut für Theorie der Kondensierten Materie, Universität Karlsruhe, 76128 Karlsruhe, Germany \\ ${ }^{2}$ Institut für Theoretische Physik, Universität zu Köln, 50937 Köln, Germany
}

\begin{abstract}
We study transport in a smooth random magnetic field, with emphasis on composite fermions (CF) near half-filling of the Landau level. When either the amplitude of the magnetic field fluctuations or its mean value $\bar{B}$ is large enough, the transport is of percolating nature. While at $\bar{B}=0$ the percolation effects enhance the conductivity $\sigma_{x x}$, increasing $\bar{B}$ (which corresponds to moving away from half-filling for the CF problem) leads to a sharp falloff of $\sigma_{x x}$ and, consequently, to the quantum localization of CFs. We demonstrate that the localization is a crucial factor in the interplay between the Shubnikov-de Haas and quantum Hall oscillations, and point out that the latter are dominant in the CF metal.
\end{abstract}

PACS numbers: 73.40.Hm, 71.10.Pm

The idea of composite fermions $(\mathrm{CF})$ [1] 3 , has been remarkably successful in accounting for the transport properties of strongly correlated two-dimensional electrons in high magnetic fields, especially near half-filling $\nu=\frac{1}{2}$ of the lowest Landau level. Within this model, each electron is replaced by a fermion carrying two flux quanta of a fictitious magnetic field oriented oppositely to the external field $\mathbf{B}_{\text {ext }}$. At half-filling, the average of this ChernSimons field cancels the external one exactly, thus offering an elegant explanation of the observed gapless state with a well-defined Fermi surface. Away from $\nu=\frac{1}{2}$, the composite fermions experience, in the mean-field sense, a net effective field $\overline{\mathbf{B}}=(1-2 \nu) \mathbf{B}_{e x t}$. An integer number $p$ of filled Landau levels of the fermions then corresponds to the fractional quantum Hall $(\mathrm{QH})$ plateau at $\nu=p /(2 p \pm 1)$. This mapping of the singularities in the strongly interacting electron system at fractional fillings onto integer $\mathrm{QH}$ transitions is a particularly attractive feature of the $\mathrm{CF}$ picture.

Confidence that the CF picture does indeed capture the essential physics of the $\nu=\frac{1}{2}$ problem is strengthened by a number of observations of Fermi-surface features at half-filling in experiments with acoustic waves [4] and in direct transport measurements [5]. In particular, in the vicinity of half-filling the dissipative resistivity $\rho_{x x}^{e}\left(B_{\text {ext }}\right)$ exhibits magnetooscillations [5] which look very much like the familiar Shubnikov-de Haas $(\mathrm{SdH})$ effect. However, there is an essential difference: the oscillations near $\nu=\frac{1}{2}$ appear to be much more strongly damped as compared to the conventional metallic phase. Generally speaking, this is in line with the fact that $\rho_{x x}^{e}$ at $\bar{B}=0$ is orders of magnitude larger than at $B_{e x t}=0$. More specifically, the enhanced damping is understood in terms of strong scattering on spatial fluctuations of the Chern-Simons field. The fluctuations of the effective magnetic field $B(\mathbf{r})=[1-2 \nu(\mathbf{r})] B_{e x t}$ around the mean value $\bar{B}$ are generated by the inhomogeneous distribution of the electron density and therefore the local filling factor $\nu(\mathbf{r})$ due to screening of the impurity potential. This mechanism of scattering dominates [2] the conductivity of the CF metal, whereas the direct scattering on the scalar random potential is of little importance.

This Letter is concerned with the disorder-induced localization of the composite fermions and its implications for transport measurements. In particular, we address the question as to the nature of the magnetooscillations near half-filling. Though it has become customary to identify the oscillations with the $\mathrm{SdH}$ effect [5], we argue that they are due to the quantum localization responsible for the QH effect. Specifically, the $\mathrm{SdH}$ and $\mathrm{QH}$ oscillations coexist with each other; what we show is that the $\mathrm{SdH}$ oscillations are much more strongly damped as compared to those induced by the quantum localization. We also demonstrate that the effective magnetic field creates a classical percolation network, which drastically suppresses the CF conductivity. It is this classical localization effect that enhances the quantum localization leading to the $\mathrm{QH}$ oscillations.

The model we deal with is that of non-interacting fermions in a random magnetic field (RMF) $\bar{B}+B(\mathbf{r})$ with the average $\bar{B}$ and the correlator $\langle B(0) B(\mathbf{r})\rangle=$ $B_{0}^{2} F(r)$. We wish to calculate the CF conductivity as a function of $\bar{B}$. The measurable electron resistivity $\rho_{\mu \nu}^{e}$ can be directly expressed in terms of the $\mathrm{CF}$ one [2]: $\rho_{x x}^{e}=\rho_{x x}, \rho_{x y}^{e}=\rho_{x y}-2 h / e^{2}$. Disorder is assumed to be created by ionized impurities randomly distributed with a sheet density $n_{i}$ (for simplicity let it be equal to the electron density $n$ ) in a thin layer separated from the electron gas by an undoped spacer of width $d \gg n^{-1 / 2}$. The correlation function of the RMF is then parameterized by $B_{0}=k_{F} / \sqrt{2} d$ and $F(r)=\left(1+r^{2} / 4 d^{2}\right)^{-3 / 2}$, where $k_{F}^{2}=4 \pi n$ and $\hbar=c=e=1$.

The RMF is characterized by two length scales: the correlation radius $d$ and the cyclotron radius in the field $B_{0}, R_{0}=k_{F} / B_{0}$. Defining the parameter $\alpha=d / R_{0}$, we can distinguish the weak-RMF regime $\alpha \ll 1$, where the mean free path $l \gg R_{0} \gg d$, and the regime of strong fluctuations $\alpha \gg 1$, where one should expect drastic de- 
viations from the Drude picture. Though $\alpha=1 / \sqrt{2}$ for the CFs at half-filling, it is instructive to get a feeling for the problem by exploring these two limiting cases. Also, in principle one can change $\alpha$ by varying the ratio of the impurity and electron densities, since $\alpha=\left(n_{i} / 2 n\right)^{1 / 2}$.

We start with the limit of weak $\bar{B}$ where the quasiclassical treatment is accurate [6]. At $\alpha \ll 1, \mathrm{CF}$ trajectories are only slightly bent on the scale of $d$, so that the Born approximation [2, 6] is valid. Accordingly, for the transport scattering time one gets $1 / \tau_{t r}=$ $\left(B_{0}^{2} / m k_{F}\right) \int_{0}^{\infty} d r F(r)=2 \alpha^{2} v_{F} / d$, where the CF effective mass $m=k_{F} / v_{F}$ is introduced. The conductivity at zero $\bar{B}$ then reads (in units of $e^{2} / h$ )

$$
\sigma_{x x}=k_{F} d / 4 \alpha^{2}, \quad \alpha \ll 1 .
$$

If the size of inhomogeneities had been strictly zero ("white noise" disorder), one would have obtained at the quasiclassical level the magnetoresistance $\Delta \rho_{x x} / \rho_{x x}=0$. To get a finite magnetoresistance, one has to include the effect of the field $\bar{B}$ on the "ballistic" scale $d$. To this end we write the equation of motion in the form $d \phi / d t=\omega_{c}+B[\mathbf{r}(t)] / m$, where $\phi$ is the angle of the velocity on the Fermi surface and $\omega_{c}=\bar{B} / \mathrm{m}$. The velocity correlation function $v_{F}^{2}\langle\cos [\phi(t)-\phi(0)]\rangle$ is then given by $v_{F}^{2}\left\langle\cos \left(\frac{1}{m} \int_{0}^{t} d t_{1} B\left[\mathbf{r}\left(t_{1}\right)\right]\right)\right\rangle$. Here $\langle\ldots\rangle$ denotes an ensemble average over exact classical trajectories $\mathbf{r}(t)$. In the limit of small $\alpha$, the classical dynamics of the system is fully chaotic (uncorrelated diffusion) and so $B[\mathbf{r}(t)]$ can be treated as a Gaussian variable with respect to the disorder averaging. Accordingly, the conductivity is

$$
\begin{aligned}
\sigma_{x x}+i \sigma_{x y} & =\frac{k_{F}^{2}}{2 m} \int_{0}^{\infty} d t e^{i \omega_{c} t-S(t)}, \\
S(t) & =\frac{1}{2 m^{2}} \int_{0}^{t} d t_{1} \int_{0}^{t} d t_{2}\left\langle B\left[\mathbf{r}\left(t_{1}\right)\right] B\left[\mathbf{r}\left(t_{2}\right)\right]\right\rangle .
\end{aligned}
$$

The double integral can be rewritten to give $S(t)=$ $\left(B_{0} / m\right)^{2} \int_{0}^{t} d t^{\prime}\left(t-t^{\prime}\right)\left\langle F\left(\left|\mathbf{r}\left(t^{\prime}\right)-\mathbf{r}(0)\right|\right)\right\rangle$. The argument of $F$ is readily expanded at $t \ll \tau_{t r}$ in powers of $\omega_{c}$ :

$$
|\mathbf{r}(t)-\mathbf{r}(0)| \simeq v_{F} t\left[1-\left(\omega_{c} t\right)^{2} / 24\right] .
$$

We can identify two different contributions to the magnetoresistance. One comes from a $\bar{B}$ dependent correction to $\tau_{t r}^{-1}=\lim _{t \rightarrow \infty} t^{-1} S(t)$. Substituting Eq. (3) into $S(t)$ we get $\tau_{t r}^{-1}(\bar{B}) \simeq \tau_{t r}^{-1}(0)\left[1+\left(d^{2} / 2 R_{c}^{2}\right) \ln (l / d)\right]$, where $R_{c}=v_{F} / \omega_{c}$ is the Larmor radius in the field $\bar{B}$. This correction would yield a positive $\Delta \rho_{x x} / \rho_{x x}$. Next, we should take into account that the relaxation kernel is not exactly the simple exponential $e^{-t / \tau_{t r}}$; specifically, $S(t) \propto t^{2}$ at $t \lesssim d / v_{F}$. This gives a 4 times larger negative contribution to the magnetoresistance, so that in total

$$
\frac{\Delta \rho_{x x}}{\rho_{x x}}=-\frac{3}{2} \frac{d^{2}}{R_{c}^{2}} \ln \frac{l}{d} .
$$

This result agrees with that derived in Ref. 7 by a different method. The sign of $\Delta \rho_{x x} / \rho_{x x}$ found for $\alpha \ll 1$ is opposite to the one observed experimentally near $\nu=\frac{1}{2}$. The discrepancy is likely due to the fact that the condition of weak RMF, $l \gg d$, is not met in the experiments.

Let us now turn to the strong-RMF regime, $\alpha \gg 1$, keeping $\bar{B}=0$. The seemingly innocent assumption about the chaotic character of the particle dynamics, which enabled us to represent the conductivity in the form (2), is not valid any more. Most particles are now out of play since they are caught in cyclotron orbits drifting along the closed lines of constant $B(\mathbf{r})$ ("van Alfvén drift"). In the adiabatic limit, their drift trajectories are periodic and so do not contribute to the conductivity. Still, however large $B_{0}$ is, there are classical paths which are not localized and percolate through the system by meandering around the lines of zero $B(\mathbf{r})$. The conductivity is determined by the particles that move along these extended "snake states" [8]. Note that there is one single percolating path on the manifold of the $B(\mathbf{r})=0$ contours; yet, the conductivity is nonzero since the snake-state trajectories form a bundle of finite width, $R_{s} \sim d / \alpha^{1 / 2}$. The conducting network is made up of those snake-states that can crossover from one critical zero- $B$ line of length $L_{s} \sim \alpha^{7 / 6} d$ (for a review of the percolation theory see Ref. 9) to another. The coupling of two adjacent percolating clusters occurs near the critical saddle-points of $B(\mathbf{r})$, which are nodes of the transport network. The characteristic distance between the nodes, i.e. the size of the elementary cell $\xi_{s}$, is then $\alpha^{2 / 3} d[9$. On length scales longer than $\xi_{s}$, the particle dynamics can be viewed as fully stochastic. We estimate the macroscopic diffusion coefficient as $D \sim \nu_{s} D_{s}$, where $\nu_{s} \sim L_{s} R_{s} / \xi_{s}^{2}$ is the fraction of particles residing in the delocalized snakestates, $D_{s} \sim \xi_{s}^{2} v_{F} / L_{s}$ their diffusion coefficient. We thus have $D \sim v_{F} R_{s}$ and, correspondingly,

$$
\sigma_{x x} \sim k_{F} d / \alpha^{1 / 2}, \quad \alpha \gtrsim 1 .
$$

It is worth noting that by comparison with the Born approximation [Eq. (1)], the conductivity is $\sim \alpha^{3 / 2}$ times larger (though the localization effects are strong and naively one might have expected the opposite). This is consistent with the experimental observation [5] that the CF conductivity at half-filling is a factor of $\sim 5$ larger than the perturbative-in- $\alpha$ result [2] $\sigma_{x x}=k_{F} d / 2$. Let us also note that $\sigma_{x x}$ given by Eq. 5 is larger by a factor of $\sim \alpha^{1 / 2}$ than that obtained for $\alpha \gg 1$ in [7] by using an "eikonal approach". The fault in [7] is not with the quasiclassical approximation itself, but with the method of disorder averaging, which is incorrect in principle at large $\alpha$; in particular, it neglects the localization of particles and the percolating character of the transport through the snake states. Whether the experimentally observed positive magnetoresistance can be understood within the percolation picture is not clear at present. 
We now consider the regime of strong $\bar{B}$, which is defined for $\alpha \sim 1$ by the condition $\bar{B} \gg B_{0}$, or equivalently, $R_{c} \ll d$. Since $d / R_{c}=k_{F} d / 2 p$, where $p$ is the number of filled CF Landau levels, and experimentally $k_{F} d \sim 10 \div 15$, the range of $p$ where the magnetooscillations are observed, $p \lesssim 7$, undoubtedly requires a strong$\bar{B}$ treatment. At $\bar{B} \gg B_{0}$, the $\mathrm{CF}$ dynamics is a slow van-Alfvén drift of the cyclotron orbits along the lines of constant $B(\mathbf{r})$, i.e. the conductivity is again determined by a percolation network of trajectories close to the $B(\mathbf{r})=0$ lines. Naively one may well think the percolation picture is very much like the one we dealt with at zero $\bar{B}$ and $\alpha \gg 1$. In actual fact, there is a crucial difference. Specifically, now there is no stochastic mixing at the nodes of the percolation network: unlike the snake states at $\bar{B}=0$, the rapidly rotating cyclotron orbits pass harmlessly through the critical saddle-points of $B(\mathbf{r})$ without changing to the adjacent cell. In the high$\bar{B}$ limit, the mixing occurs on the links of the network and is only due to the weak scattering between the drift trajectories.

In order to calculate the conductivity at $\bar{B} \gg B_{0}$, we first need to integrate out the fast cyclotron rotation, taking care not to lose the effect of the non-adiabatic mixing. A similar question for the electron system in a random scalar potential was recently addressed in [10]. We write the equation of motion in the form

$$
z(t)=i v_{F} \int_{0}^{t} d t^{\prime} e^{i \int_{0}^{t^{\prime}} d t^{\prime \prime} \Omega\left[z\left(t^{\prime \prime}\right)\right]}
$$

where $z(t)=x+i y$ stands for the exact trajectory $\mathbf{r}(t)=$ $(x, y)$ in the field $\bar{B}+B(z)$, and $\Omega(z)=\omega_{c}+B(z) / m$. It is convenient to introduce the guiding center coordinate $\zeta(t)=z(t)-R_{c}[\zeta(t)] e^{i \varphi(t)}$, where $R_{c}$ is the local cyclotron radius at the point $\zeta(t)$ of the drift trajectory and $\varphi(t)=\int_{0}^{t} d t^{\prime} \Omega_{c}\left[\zeta\left(t^{\prime}\right)\right]$ is expressed in terms of the local cyclotron frequency. The velocity of the guiding center is given by $\dot{\zeta}(t)=i v_{F}\left(e^{i \int_{0}^{t} d t^{\prime} \Omega\left[z\left(t^{\prime}\right)\right]}-e^{i \varphi(t)}\right)-\dot{R}_{c} e^{i \varphi}$. After averaging over the cyclotron motion (denoted by $\langle\ldots\rangle_{c}$ ), the first term leads to the usual expression for the drift velocity $\mathbf{v}_{d}=\langle\dot{\zeta}\rangle_{c}=\left[m v_{F}^{2} / 2(\bar{B}+B)^{3}\right](\nabla B \times(\overline{\mathbf{B}}+\mathbf{B}))$. At this level the drift occurs strictly along the lines of constant $B(\mathbf{r})$, so that the conductivity would be zero. The time dependence of the cyclotron radius in the second term gives rise to the leading non-adiabatic contribution $\delta \mathbf{v}_{d}=\left\langle\left(2 v_{d}^{2} / v_{F}\right) e^{i \varphi}\right\rangle_{c}$, allowing the particle to perform a random walk around the $B(\mathbf{r})=0$ contour. Because of the rapidly oscillating factor $e^{i \varphi}, \delta \mathbf{v}_{d}$ will be exponentially small. We define the diffusion coefficient associated with the motion perpendicular to the drift trajectory as $D_{\perp}=\frac{1}{4} \lim _{t \rightarrow \infty} t^{-1}\left\langle\left[\int \delta \mathbf{v}_{d}(t) d t\right]^{2}\right\rangle_{d} \propto e^{-W}$, where $\langle\ldots\rangle_{d}$ denotes the average over the impurity ensemble along the drift lines.

Expressing the phase $\varphi$ by integration along the contour, $\varphi=\omega_{c} \int d l v_{d}^{-1}(l)$, and shifting the integration into the complex plane one observes that the exponent $W$ is determined by the phase $\varphi$ picked up at the singular point of the correlator $\langle B(0) B(r)\rangle$ at $r=2 i d$. Consequently,

$$
W=-\ln \left\langle e^{i \omega_{c} \int_{0}^{2 i d} d v_{d}^{-1}(l)}\right\rangle
$$

where the integration should be done along the straight line connecting the points $l=0$ and $l=2 i d$. It follows that the dominant contribution to $D_{\perp}$ comes from rare fluctuations in which the drift velocity greatly exceeds the typical value $v_{F}\left(B_{0} / \bar{B}\right)^{2}$. Indeed, $W$ can be written as a sum of two terms,

$$
W_{1}=\frac{1}{2} \int \frac{d^{2} \mathbf{k}}{(2 \pi)^{2}} \frac{\left|v_{d x_{\mathbf{k}}}^{0}\right|^{2}}{\left\langle v_{d x} v_{d x}\right\rangle_{\mathbf{k}}}, W_{2}=i \omega_{c} \int_{0}^{2 i d} \frac{d x}{v_{d x}^{0}(x, 0)} .
$$

Here $W_{1}$ represents the probability for the optimum fluctuation $v_{d x}^{0}(\mathbf{r})$ to occur, $W_{2}$ stands for the probability of the non-adiabatic scattering on this fluctuation. Solution of the variational equation $\delta W / \delta v_{d x}^{0}=0$ then yields $v_{d x}^{0}(\mathbf{r})=v_{F}\left(B_{0} / \bar{B}\right) \mathcal{F}(\mathbf{r} / d)$, where $\mathcal{F}$ is a dimensionless function. We thus get

$$
W=c\left(\bar{B} / B_{0}\right)^{2},
$$

where the numerical coefficient $c \sim 1$. Choosing $v_{d x}^{0}(\mathbf{r}) \propto$ $\left\langle v_{d x}(0) v_{d x}(\mathbf{r})\right\rangle$ as a trial function with the variational parameter $v_{d x}^{0}(0)$, we obtain the estimate $c \simeq 1.6$.

The non-adiabatic mixing of drift trajectories yields the conductivity $\sigma_{x x} \sim k_{F} \delta$, where $\delta$ is an effective width of the links of the percolation network. This $\delta$ obeys the equation $\delta^{2} \sim D_{\perp} L(\delta) / v_{d}$, which is the condition of connectivity of the network. Here $L(\delta) \sim d(d / \delta)^{7 / 3}$ is the characteristic perimeter of the cells [9]. Consequently,

$$
\sigma_{x x}=k_{F} d \times f\left(\frac{\bar{B}}{B_{0}}\right) \exp \left[-\frac{3 c}{13}\left(\frac{\bar{B}}{B_{0}}\right)^{2}\right],
$$

where $f(x)$ is a power-law function. The conductivity is seen to fall off sharply beyond the scale $\bar{B} \sim B_{0}$. This unusual manifestation of classical localization is also found in non-interacting systems with a scalar random potential [10]. Finally, it is worth noting that, however sophisticated the dissipative conductivity network may be, the macroscopic Hall conductivity $\sigma_{x y}$ in the metallic system at $\bar{B} \gg B_{0}$ assumes the collisionless form $\sigma_{x y}=2 \pi n / \bar{B}=p \gg \sigma_{x x}$ 11.

Having found the monotonic component of $\sigma_{x x}(\bar{B})$, we turn to the magnetooscillations. These are conventionally interpreted as the $\mathrm{SdH}$ effect for the CFs. We want to compute the disorder-induced Dingle factor of the

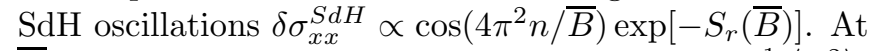
$\bar{B} \gg B_{0}$ the damping exponent is given by $S_{r}=\frac{1}{2}\left\langle\Phi^{2}\right\rangle$, where $\Phi$ is the random- $B$ flux through the cyclotron orbit in the field $\bar{B}[6]$. Had the CF dynamics been fully 
chaotic, $\langle\ldots\rangle$ here would have meant a disorder average over all possible configurations of $B(\mathbf{r})$, which yields $S_{r}=4 \pi^{2} p^{4} /\left(k_{F} d\right)^{2}[6]$. The result might look puzzling, since this $S_{r}$ is numerically by far larger [6] than what is observed experimentally. However, in the limit $\bar{B} \gg B_{0}$, only the particles that drift along the percolating trajectories with zero $B(\mathbf{r})$ contribute to $\sigma_{x x}$. Accordingly, whereas the above $S_{r}$ describes the damping of the total density of states, the averaging we need to do should be performed along the contours $B(\mathbf{r})=0$. The result is

$$
S_{r}=\left(21 \pi^{2} / 4\right) p^{8} /\left(k_{F} d\right)^{6} .
$$

Both the extremely sharp falloff of the amplitude of the oscillations with increasing $p$ and the numerical value of $S_{r}$ are in fair agreement with the experimental data available to date [5]; yet, there are good reasons to question this picture. The point is that the CF system, like a conventional metal, will exhibit magnetooscillations even if those of the density of states are totally neglected. Specifically, in the diffusive regime, the quantum interference of scattered waves leads to the $\mathrm{QH}$ oscillations [12] $\delta \sigma_{x x}^{Q H} \propto \cos \left(2 \pi \sigma_{x y}\right) \exp \left[-S_{l}(\bar{B})\right]$, where

$$
S_{l}=2 \pi \sigma_{x x} .
$$

The confusion that one might have at this point is over the notion that the QH effect transforms into the SdH oscillations with decreasing magnetic field, which is manifestly not true. Likewise, let us stress that the observation of the $\mathrm{QH}$ oscillations at $p \gg 1$ does not require exponentially low temperatures, - while the characteristic localization length does grow exponentially fast with $p$, the oscillations come from the interference on the small scale of the effective mean free path. Note that the periods of the $\mathrm{QH}$ and $\mathrm{SdH}$ oscillations coincide at $\bar{B} \gg B_{0}$, but the damping factors are very different. The quasiclassical approach which led us to the exponential falloff of $\sigma_{x x}$ with decreasing $p$ fails and the QH effect shows up at $p^{Q H} \sim k_{F} d / \ln ^{1 / 2}\left(k_{F} d\right)$, where $\sigma_{x x}$ drops to unity. At $p \lesssim p^{Q H}$ the oscillations are due to the $\mathrm{QH}$ effect. This picture is in good agreement with experiment: from the typical $\rho_{x x} \simeq 0.02 \div 0.03$ at $p \simeq 6 \div 7$, where the first resistivity minimum is observed, one finds $\sigma_{x x} \simeq \rho_{x x} p^{2} \simeq 1 \div 1.5$. Furthermore, at smaller $p \leq 5$, the values of $\sigma_{x x}$ at the maxima are close to $\frac{1}{2}$, thus confirming that the $\mathrm{CFs}$ are indeed in the $\mathrm{QH}$ regime. As for the SdH oscillations, Eq. (11) tells us that they might only be observable at $p \lesssim p^{S d H} \sim\left(k_{F} d\right)^{3 / 4} \ll p^{Q H}$. The last inequality means that in the limit $k_{F} d \gg 1$ there is no room for the SdH effect. In practical terms, however, $p^{Q H}$ and $p^{S d H}$ are numerically close at the experimentally relevant $k_{F} d \sim 10 \div 15$, so that the $\mathrm{SdH}$ effect still can contribute to the first couple of minima $(p=7,6)$.

In conclusion, we have studied the fermion kinetics in a smoothly varying RMF with mean $\bar{B}$. We calcu- lated the conductivity in the regime of strong RMF and zero $\bar{B}$, when the transport is determined by percolating snake-states. We demonstrated that increasing $\bar{B}$ leads to the classical localization of fermions, the key signature of which is the exponentially sharp drop in $\sigma_{x x}$. We showed that this yields a strong enhancement of the magnetooscillations, thus explaining why they are observed at large $p$, where the $\mathrm{SdH}$ oscillations are negligible. We analyzed the interplay between the $\mathrm{SdH}$ and $\mathrm{QH}$ oscillations and argued that the latter are dominant in the limit $k_{F} d \gg 1$.

We are grateful to I. Aleiner, B. Altshuler, J. Hajdu, L. Rokhinson, A. Shelankov, H. Stormer, and D. Tsui for interesting discussions. This work was supported by the Deutsche Forschungsgemeinschaft (in part through SFB195), by the German-Israeli Foundation, and by the Russian Foundation for Basic Research under Grant No. 96-02-17894a.

* Also at St.Petersburg Nuclear Physics Institute, 188350 St.Petersburg, Russia.

$\dagger \quad$ Also at A.F.Ioffe Physico-Technical Institute, 194021 St.Petersburg, Russia.

[1] D. K. Jain, Phys. Rev. Lett. 63, 199 (1989); Phys. Rev. B 40, 8079 (1989); 41, 7653 (1990).

[2] B. I. Halperin, P. A. Lee, and N. Read, Phys. Rev. B 47, 7312 (1993).

[3] A. Lopez and E. Fradkin, Phys. Rev. B 44, 5246 (1991); 47, 7080 (1993).

[4] R. L. Willett, K. W. West, and L. N. Pfeiffer, Phys. Rev. Lett. 75, 2988 (1995).

[5] R. R. Du el al., Solid State Commun. 90, 71 (1994); Phys. Rev. Lett. 70, 2944 (1993); D. R. Leadley et al., Phys. Rev. Lett. 72, 1906 (1994); Phys. Rev. B 53, 2057 (1996); P. T. Coleridge et al., Phys. Rev. B 52, R11603 (1995); L. P. Rokhinson and V. J. Goldman, Report No. cond-mat/9704043.

[6] A. G. Aronov, E. Altshuler, A. D. Mirlin, and P. Wölfle, Phys. Rev. B 52, 4708 (1995); A. D. Mirlin, E. Altshuler, and P. Wölfle, Ann. Phys. (Leipzig) 5, 281 (1996).

[7] D. V. Khveshchenko, Phys. Rev. Lett. 77, 1817 (1996).

[8] D. B. Chklovskii and P. A. Lee, Phys. Rev. B 48, 18060 (1993); D. K. K. Lee, J. T. Chalker, and D. Y. K. Ko, Phys. Rev. B 50, 5272 (1995).

[9] M. B. Isichenko, Rev. Mod. Phys. 64, 961 (1992).

[10] M. M. Fogler, A. Yu. Dobin, V. I. Perel, and B. I. Shklovskii, Report No. cond-mat/9702121.

[11] S. A. Trugman, Phys. Rev. B 27, 7539 (1983).

[12] A. M. M. Pruisken, in The Quantum Hall Effect, edited by R. E. Prange and S. M. Girvin (Springer, Berlin, 1990). 